\title{
Caracterización del consumo de larvas de culícidos (Diptera) en dos especies de peces indígenas de la zona central de Argentina
}

\author{
Alejo F. Bonifacio, Ma. Liliana Aun \& Ricardo A. Martori \\ Departamento de Ciencias Naturales, Facultad de Ciencias Exactas, Físico-Químicas y Naturales, Universidad Nacional de Río Cuarto (UNRC), Ruta 36, Km 601, 5800, Río Cuarto, Argentina. \\ (alejobonifacio@gmail.com)
}

\begin{abstract}
Characterization of Culicidae larvae (Diptera) consumption in two species of native fish in the central zone of Argentina. Mosquitoes represent a threat to human health and animals because they act as vectors of several diseases. Native fish species are potential candidates to be considered for biological control populations of mosquitoes. Tests of half-hour and 24-hour consumption of Culex pipiens (Linnaeus, 1758) larvae by Cnesterodon decemmaculatus (Jenyns, 1842) and Jenynsia multidentata (Jenyns, 1842) were carried out, measuring the standard length, mouth width and weight in individuals of the two species of fishes. In both tests J. multidentata consumed more C. pipiens than C. decemmaculatus, being $C$. decemmaculatus female consumption higher than male consumption (and equal than both sexes of $J$. multidentata in the 24-hour test). These results remained unchanged when making comparisons of absolute or relative consumption in the half-hour test, but when consumptions relative to weight were compared no difference between species was found in the 24-hour test. Regression analysis between morphometric variables and weight vs. consumption rates showed low explanatory value for the half-hour test, while in the 24-hour test these variables had higher explanatory value, especially for the mouth width. Finally, a half-hour test was performed over $C$. decemmaculatus females using C. pipiens and Aedes aegypti (Linnaeus, 1742) larvae and it showed a strong preference for the latter ones. This work allowed us to observe that there are large differences in consumption rates of $C$. pipiens larvae between the fish species under study in short periods. Differences are attenuated when consumption times are longer and may even disappear when weight is taken into account.
\end{abstract}

KEYWORDS. Cnesterodon decemmaculatus, Jenynsia multidentata, biocontrol, mosquito larvae.

RESUMEN. Los mosquitos representan una amenaza para la salud del hombre y de los animales debido a que actúan como vectores de distintas enfermedades. Especies de peces nativos son potenciales candidatos a tenerse en cuenta para control biológico de poblaciones de culícidos. Experiencias de consumo de media hora y de 24 horas por Cnesterodon decemmaculatus (Jenyns, 1842) y Jenynsia multidentata (Jenyns, 1842) se llevaron a cabo con larvas de Culex pipiens (Linnaeus, 1758), midiéndose longitud estándar, ancho de boca y peso en individuos de las dos especies. En ambas pruebas, J. multidentata consumió más $C$. pipiens que $C$. decemmaculatus, consumiendo las hembras de esta última especie más que los machos (e igual a ambos sexos de $J$. multidentata en la prueba de 24 horas de duración). Estos resultados no variaron cuando se compararon tantos consumos absolutos o relativos para las pruebas de media hora, sin embargo cuando se compararon los consumos relativos al peso no se encontraron diferencias entre las especies para las pruebas de 24 horas. Análisis de regresión entre las tasas de consumo versus las variables morfométricas y el peso mostraron poco valor explicativo en las pruebas de media hora de duración, mientras que en las pruebas de 24 horas de duración los análisis de regresión tuvieron un mayor valor explicativo, especialmente con el ancho de la boca. Por último, pruebas de media hora de duración fueron llevadas a cabo exponiendo a hembras de $C$. decemmaculatus con larvas de $C$. pipiens y Aedes aegypti (Linnaeus, 1742) observándose una fuerte preferencia por las últimas. Este trabajo permitió evidenciar que las especies de peces en estudio presentan grandes diferencias en las tasas de consumo de C. pipiens en periodos cortos. Estas diferencias se atenuaron cuando las tasas de consumo se prolongaron y hasta llegar a desaparecer cuando el peso se tuvo en cuenta.

PALABRAS-CLAVE. Cnesterodon decemmaculatus; Jenynsia multidentata, control biológico, larvas de culícidos.

Los mosquitos representan una amenaza para la salud del hombre y de los animales debido a que actúan como vectores de distintas enfermedades. Provocan disminución en el rinde de la producción pecuaria y desalientan al hombre en la realización de actividades recreativas al aire libre (GIRI \& CoLLINGS, 2003).

En la zona central de Argentina se encuentran especies de culícidos urbanos tales como Aedes aegypti (Linnaeus, 1742) y Culex pipiens (Linnaeus, 1758) siendo la primera vector del virus del Dengue, y la segunda vector de la Encefalitis de San Luis (Rossi et al., 2002). Estas especies son típicas de ambientes urbanos en varias partes del mundo, pudiendo criarse en recipientes artificiales de variados tamaños (Vezzani \& Albicócco, 2009). Culex pipiens, además coloniza ambientes naturales lénticos de variados tipos y es la especie de culícido predominante en la zona central de Argentina, que es donde se realizó el estudio.
Entre los métodos utilizados para el control de mosquitos se encuentra el uso de pesticidas químicos, estos pueden ser dañinos para la salud humana y tener efectos nocivos para las demás poblaciones de organismos con los que comparten hábitat, incluyendo a sus depredadores naturales. Además, aumentan la probabilidad de que se genere resistencia al insecticida. Estas razones llevan a que se exploren métodos alternativos que lleven a un mejor control de las poblaciones de mosquitos (CHANDRA et al., 2010), siendo el control biológico una opción que puede llegar a ser más efectiva si se realiza teniendo el suficiente conocimiento de base para evitar sus posibles perjuicios. El agente de control biológico más ampliamente usado es el "pez mosquito" del oeste Gambusia affinis (Baird \& Girard, 1853) y el "pez mosquito" del este G. holbrooki (Girard, 1859) (KumAR \& HwANG, 2005) ambos de la familia Poeciliidae. Sin embargo, han demostrado comportarse 
como exitosos invasores en numerosos lugares en donde han sido introducidos (GAMradT \& KaTs, 1996; HowE et al., 1997; GoOdsell \& Kats, 1999; LeYSe et al., 2004; Ayala et al., 2007; Rowe et al., 2007).

Como alternativa a esta especie y a sus posibles efectos negativos, se encuentran como integrantes de la fauna nativa de la zona central de la Argentina y de amplia distribución en el sur de Sudamérica (LóPEZ et al., 2008) a Cnesterodon decemmaculatus (Jenyns, 1842) (Poecilidae) y Jenynsia multidentata (Jenyns, 1842) (Anablepidae), las cuales son habitantes muy frecuentes en cuerpos de agua poco profundos, tienen una estrategia reproductiva explosiva y sus poblaciones crecen a gran velocidad cuando las condiciones son propicias. Como son especies muy resistentes a la degradación de la calidad del agua, habitualmente son dominantes en las comunidades de peces en ambientes alterados (BISTONI et al., 1999; SCASSO et al., 2001; Hued \& Bistoni, 2005).

Con respecto a $C$. decemmaculatus y $J$. multidentata existen pocos trabajos que evalúen su potencialidad en el consumo de larvas de culícidos y los resultados obtenidos en laboratorio y de campo son algo contradictorios.

Sobre $C$. decemmaculatus estudios de preferencia de dieta en laboratorio y de dieta en ambientes naturales desalientan su uso como controladores biológicos (QUINTANS et al., 2009, 2010); contrariamente, estudios llevados a cabo en canales urbanos y en laboratorio apoyan su utilización para estos fines (MARTI et al., 2006; TRANCHIDA et al., 2010). Jenynsia multidentata posee menos antecedentes que la especie anterior. Sin embargo, estudios llevados a cabo tanto en laboratorio como en ambientes naturales apoyan su uso como controlador biológico de poblaciones de mosquitos (Ringuelet et al., 1967; MarTi et al., 2006; Quintans et al., 2009). Por último, J. multidentata posee un nivel trófico superior al de $C$. decemmaculatus (LÓPEZ CAzorla et al., 2003; Quintans et al., 2009). Frente a estos antecedentes la hipótesis planteó que $J$. multidentata al pertenecer a un nivel trófico superior a manifestaría una mayor avidez y tasa de consumo de larvas de C. pipiens que $C$. decemmaculatus.

El objetivo del presente trabajo fue evaluar, en ensayos de laboratorio, el consumo de larvas de culícidos por parte de Cnesterodon decemmaculatus y Jenynsia multidentata en relación a variables morfométricas y peso, con el fin de estimar su potencial como controladores biológicos de mosquitos.

\section{MATERIALES Y MÉTODOS}

Recolección y mantenimiento de larvas de mosquito y peces. Los experimentos se llevaron a cabo en laboratorio con individuos de Cnesterodon decemmaculatus y Jenynsia multidentata recolectados en la desembocadura de los desagües pluviales de la ciudad de Río Cuarto, Córdoba ( $33^{\circ} 08^{\prime} 28^{\prime \prime}$ 'S; $64^{\circ} 17^{\prime} 05^{\prime \prime}$ O). Para la colecta se utilizó una red de mano con una abertura de $30 \mathrm{~cm}$ por $50 \mathrm{~cm}$ y con una luz de malla de $2 \mathrm{~mm}$. Los peces fueron trasladados al laboratorio y mantenidos para su aclimatación en acuarios de 15 litros con agua declorada, y aireados con una bomba que insuflaba aire durante una semana.

Para las experiencias se utilizaron larvas de culícidos del estadio 4 que fueron colectadas de depósitos naturales del campus de la Universidad Nacional de Río Cuarto y de depósitos artificiales de la Ciudad de Río Cuarto.

Diseño de la experiencia en laboratorio. Se realizaron experiencias de tasa de consumo con machos y hembras de $C$. decemmaculatus y $J$. multidentata de 30 minutos $(\mathrm{n}=80)$ y de 24 horas $(\mathrm{n}=52)$ de duración. Todas las experiencias se llevaron a cabo con un pez por recipiente. Las primeras se llevaron a cabo en recipientes con $200 \mathrm{ml}$ de agua declorada y 30 larvas de C. pipiens $(\mathrm{n}=20$ para cada sexo de cada especie), y las segundas en recipientes con $1500 \mathrm{ml}$ de agua declorada y 150 larvas C. pipiens ( $\mathrm{n}=13$ para cada sexo de cada especie), para tener en cuenta de esta manera diferencias en voracidad de ambas especies de peces. Se realizó un tercer tipo de experiencia, consistente en determinar la preferencia por especie de larva, durante 30 minutos, en $300 \mathrm{ml}$ de agua declorada, ofreciendo 20 larvas de C. pipiens y 20 larvas de Aedes aegypti y utilizando hembras de $C$. decemmaculatus $(\mathrm{n}=20)$. Al final de cada experiencia se contaron las larvas remanentes para conocer el número de consumidas.

Cada pez fue pesado en una balanza electrónica (precisión: $0,001 \mathrm{~g}$ ) y se midió su longitud estándar (LS) $\mathrm{y}$ ancho de boca $(\mathrm{AB})$ usando un calibre digital con una precisión de $0,01 \mathrm{~mm}$, estas medidas permitieron dividir el consumo de cada pez por su peso, su longitud estándar y ancho de boca, de esta manera se ponderaron las diferencias entre individuos y especies. Las mediciones se realizaron después de cada experiencia para evitar que la manipulación de los individuos afecte las tasas de consumo de los peces. Se realizó una corrección de los pesos medidos restándole el peso equivalente al total de larvas que consumió cada pez. Para estimar el peso de una larva se pesó una muestra de 50 larvas de estadio 4 escurridas, con el fin de disminuir el error. En el caso de las experiencias de 24 horas no se corrigió el peso, ya que el tiempo de digestión medio de ambas especies es de alrededor de $6 \mathrm{~h}$ (MARTI et al., 2006), que es un tiempo considerablemente menor al que duró esta experiencia, por lo que gran parte de las larvas consumidas al final de cada experiencia podrían haber sido defecadas; debido a esto se supuso que todos los peces tenían un grado similar de repleción en sus tractos digestivos.

Tratamiento estadístico. A través del análisis de la varianza no paramétrica de Kruskal-Wallis se comparó, para las dos primeras experiencias, el consumo absoluto y el consumo relativizado con respecto a tamaño y peso. La relativización se realizó con el objetivo de eliminar el efecto de las variables mencionadas sobre las unidades experimentales. Además, se realizaron análisis de regresión simple discriminando por sexo y especie entre los consumos de larvas de C. pipiens (variable respuesta) y tomando como variables regresoras a las medidas morfométricas y el peso. Estas regresiones se realizaron con los resultados 
de las experiencias de 30 minutos y las de 24 horas de duración. Se graficaron aquellas regresiones que fueron significativas $(\mathrm{p}<0,05)$ y con valores de $\mathrm{R}^{2}$ mayores al 0.50 .

Para la experiencia de preferencia de larvas se llevó a cabo una prueba $\mathrm{T}$ para muestras apareadas. Este análisis evalúa si la media de las diferencias en el consumo de cada una de las especies de larvas es diferente de cero.

\section{RESULTADOS}

En la experiencia de consumo en 30 minutos se encontraron diferencias significativas en los consumos de larvas entre las especies teniendo o sin tener en cuenta el factor sexo $(p<0.0001)$. Teniendo en cuenta el factor sexo, tanto en el consumo absoluto (Fig. 1) como en los relativos a las variables morfométricas y al peso, la menor tasa de consumo fue registrada para el tratamiento $C$. decemmaculatus macho seguido por $C$. decemmaculatus hembra. La mayor tasa de consumo correspondió a la especie $J$. multidentata, no registrándose diferencias significativas entre machos y hembras. Comparando solamente el efecto de la especie se observó un consumo significativamente mayor $(\mathrm{p}<0.0001)$ en $J$. multidentata, tanto en consumo absoluto como relativo a cualquiera de las variables morfométricas o el peso.

En la experiencia de consumo en 24 horas teniendo en cuenta el factor sexo, tanto en el consumo absoluto (Fig. 2) como en los relativos a las medidas morfométricas $(p<0.0001)$ y el peso $(p<0.0018)$, la menor tasa de consumo fue registrada para el tratamiento $C$. decemmaculatus macho mientras que la mayor tasa correspondió a ambos sexos de $J$. multidentata y las hembras de $C$. decemmaculatus, no existiendo diferencias significativas entre éstos últimos. Comparando solamente el efecto de la especie se observó que el consumo absoluto como el consumo relativo a las variables morfométricas fue significativamente mayor para $J$. multidentata $(\mathrm{p}=0.02)$. En contraposición con las comparaciones anteriores, no se encontraron diferencias entre las especies en el consumo en relación al peso de los individuos ( $\mathrm{p}=0.15$ ) (Fig. 3).

Los análisis de regresión para la prueba de consumo en 30 minutos para cada especie y sexo versus variable morfométrica en particular o peso resultaron siempre en valores de $\mathrm{R}^{2}$ inferiores a $0.50 \mathrm{y} / \mathrm{o} \mathrm{p} \geq 0.05$.

Los análisis de regresión para la prueba de consumo diario para cada especie y sexo versus variable morfométrica en particular o peso, presentaron valores de $\mathrm{R}^{2}$ mayores a 0.50 , y fueron significativos en cuatro casos. Uno de estos fue la de regresión entre la longitud estándar y el consumo para los machos de $C$. decemmaculatus $\left(\mathrm{R}^{2}=0.6079\right.$ y $\mathrm{p}=$ 0.0017) (Fig. 4). Los restantes tres fueron entre el ancho de la boca y consumo para machos $\left(\mathrm{R}^{2}=5879 \mathrm{y} \mathrm{p}=0.0022\right)$ y hembras $\left(\mathrm{R}^{2}=0.7220 \mathrm{y} \mathrm{p}=0.0002\right)$ de $J$. multidentata y las hembras de $C$. decemmaculatus $\left(\mathrm{R}^{2}: 0.5659\right.$ y $\left.\mathrm{p}=0.003\right)$ (Figs 5, 6 y 7, respectivamente).

La prueba de preferencia entre larvas de A. aegypti y $C$. pipiens presentó como resultado una diferencia significativa entre el consumo de estos dos ítems $(\mathrm{p}<0.0001)$ siendo mayor el consumo de larvas de A. aegypti. En ninguno de los individuos utilizados en la experiencia el consumo de larvas de $C$. pipiens fue mayor que el de larvas de $A$. aegypti (Fig. 8). El consumo promedio de larvas de $A$. aegypti fue de un $88 \%$, ampliamente superior al registrado para larvas de C. pipiens (12\%).

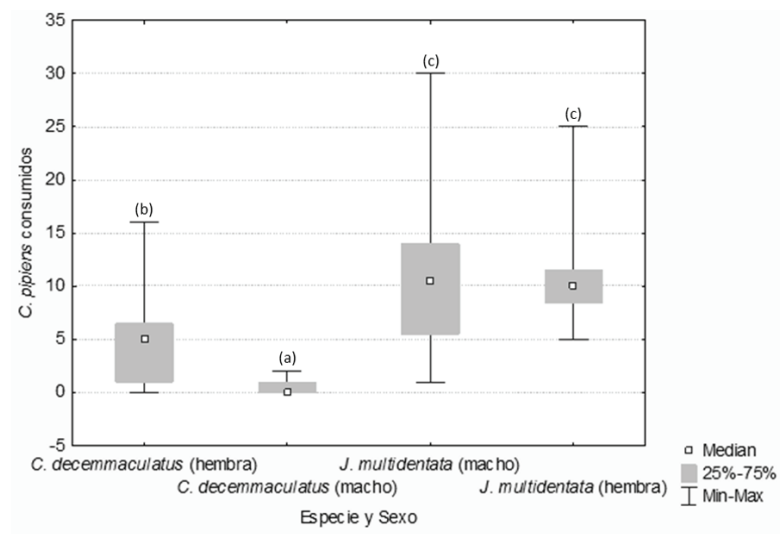

Fig. 1. Gráfico de caja que muestra la distribución de los consumos de Culex pipiens (Linnaeus, 1758) para cada especie y sexo. Letras diferentes indican diferencias significativas entre grupos $(\mathrm{p}<0.05)$.

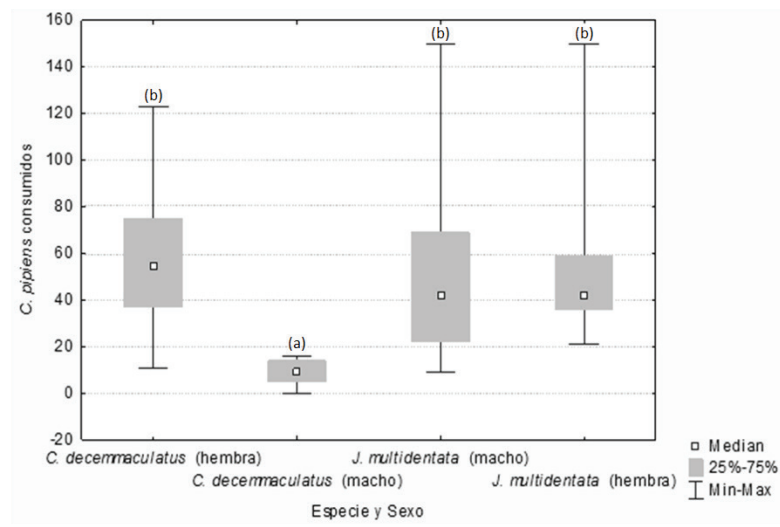

Fig. 2. Gráfico de caja que muestra la distribución de los consumos de Culex pipiens (Linnaeus, 1758) para cada especie y sexo. Letras diferentes indican diferencias significativas entre grupos $(\mathrm{p}<0.05)$.

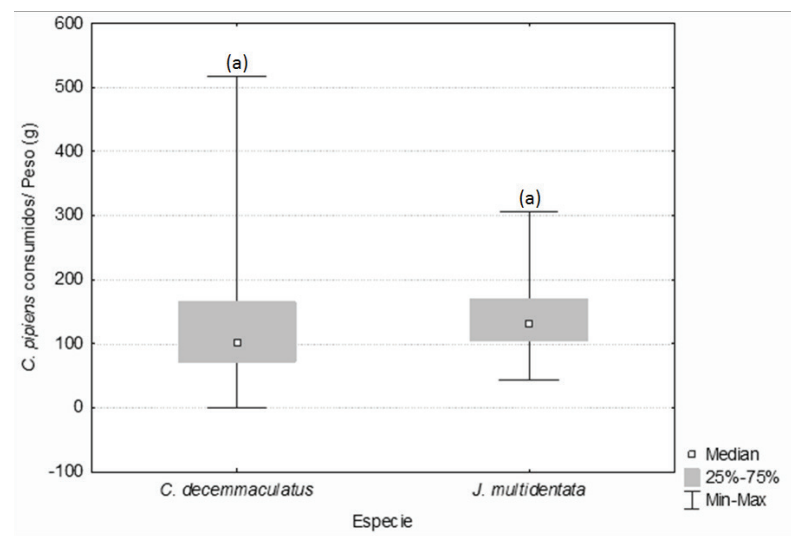

Fig. 3. Gráfico de caja que muestra la distribución de los consumos de Culex pipiens (Linnaeus, 1758) para cada especie ponderado con el peso. Letras diferentes indican diferencias significativas entre grupos $(\mathrm{p}<0.05)$. 


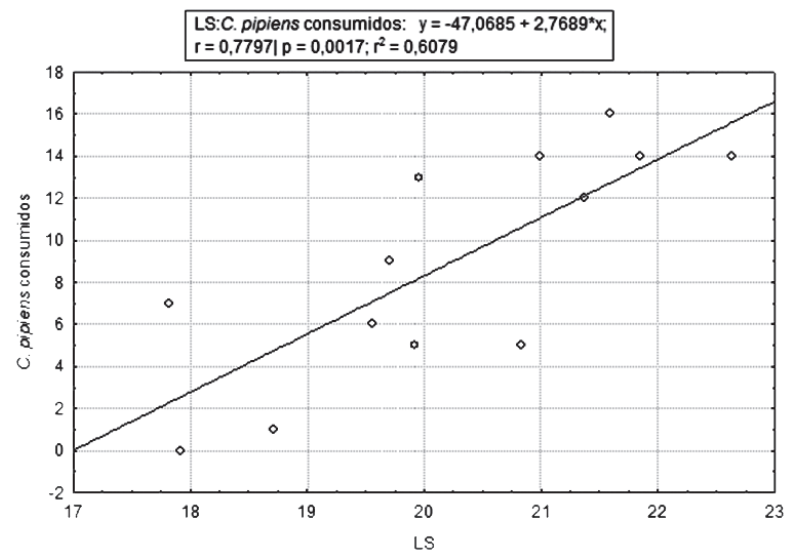

Fig. 4. Recta de regresión entre el consumo de larvas en 24 horas y el largo estándar (LS) para los machos de C. decemmaculatus.

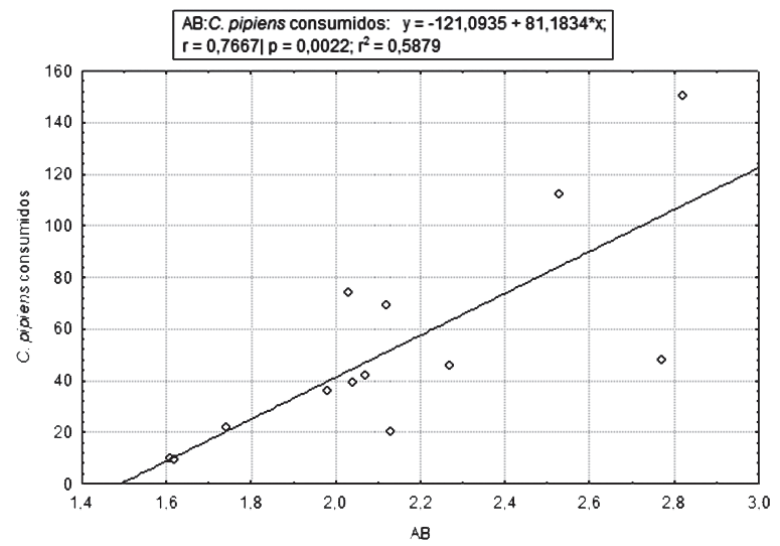

Fig. 5. Recta de regresión entre el consumo de larvas en 24 horas y el ancho de boca (AB) para los machos de J. multidentata.

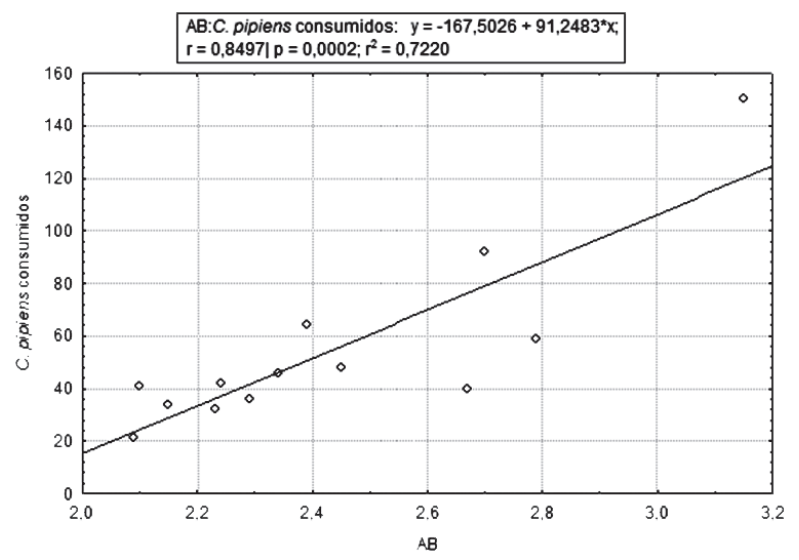

Fig. 6. Recta de regresión entre el consumo de larvas en 24 horas y el ancho de boca (AB) para las hembras de J. multidentata.

\section{DISCUSIÓN}

En el presente trabajo, la comparación de las tasas de consumo de larvas de C. pipiens indican que $J$. multidentata posee tasas significativamente mayores con respecto a las tasas registradas para ambos sexos de C. decemmaculatus en ensayos de 30 minutos de duración y para machos de

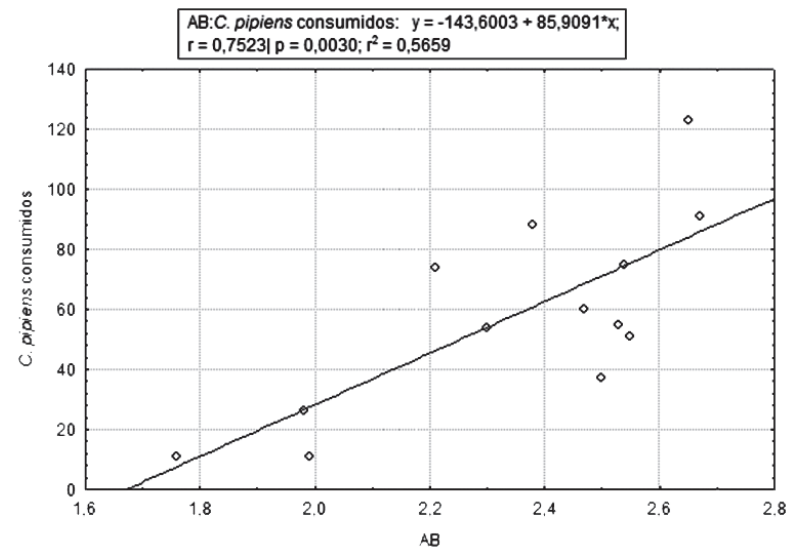

Fig. 7. Recta de regresión ente el consumo de larvas en 24 horas y el ancho de boca (AB) para las hembras de C. decemmaculatus.

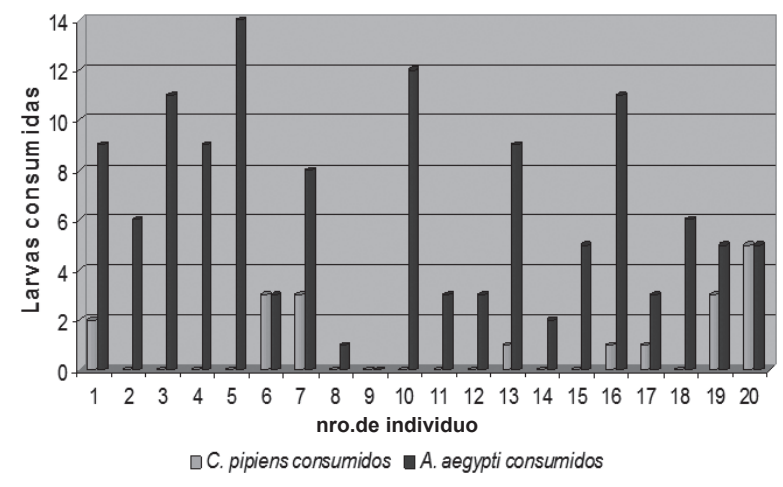

Fig. 8. Gráfico de barras que denota el consumo de cada especie de larva para cada uno de los individuos de la experiencia.

C. decemmaculatus en los ensayos de 24 horas de duración; cuando se compararon especies y sexos conjuntamente. Cuando la comparación fue entre especies solamente el consumo de larvas de $C$. pipiens en casi todos los casos fue significativamente mayor para J. multidentata; solamente ponderando el consumo con el peso no se encontraron diferencias significativas entre las mismas en los ensayos de 24 horas de duración. Vale decir, que el peso es la variable que tiene la mayor importancia a nivel de control biológico, ya que en cualquier ambiente en el que se introduzcan peces con este fin, este sostendrá una biomasa determinada de individuos, independientemente del largo o el ancho de boca que presenten. Por estos resultados, no podemos apoyar completamente la hipótesis inicial que postulaba a $J$. multidentata como mayor consumidora de larvas de $C$. pipiens, la voracidad en el caso de estas especies no sería una buena indicadora de la capacidad final de consumo de larvas, por lo menos en estas especies de peces. Por otro lado, es necesario destacar que se evaluó el consumo de larvas del $4^{\circ}$ estadío, que son lo suficientemente grandes como para ocasionar un problema mecánico para la depredación por parte de los machos de $C$. decemmaculatus. Lo mencionado podría subestimar la capacidad controladora de éstos sobre larvas de los primeros estadios. 
La importancia del ancho de boca en las regresiones de las experiencias de 24 horas demostraría una relación inversa entre los tiempos de manipulación de las presas y los tamaños de boca, por lo que el tamaño de la boca limitaría el consumo de larvas en mayor medida que las demás variables.

Los resultados de este trabajo apoyan y complementan los obtenidos por MARTI et al. (2006) al incluir en las experiencias a los machos de ambas especies $\mathrm{y}$ al tener en cuenta un amplio rango de tamaños. QUINTANS et al. (2010) muestra una preferencia de $C$. decemmaculatus hacia cladóceros y otros ítems alimenticios siendo las larvas de C. pipiens el ítem menos preferido, por lo que no recomienda a esta especie para el control biológico de larvas de mosquitos. Además Quintans et al. (2009) no registró presencia de larvas de culícidos en los tractos digestivos de C. decemmaculatus, mientras que si estuvieron presentes en los tractos de $J$. multidentata, en estudios realizados a campo. Estos dos últimos trabajos dan más apoyo a $J$. multidentata que $C$. decemmaculatus como agente de control biológico. Sin embargo, MARTI et al. (2006) y TRANCHIDA et al. (2010) comprobaron como la liberación de $C$. decemmaculatus en ambientes artificiales lleva a la desaparición de larvas de culícidos en el plazos que van desde 4 a 22 semanas dependiendo de la densidad de introducción de esta especie.

Las tasas de consumo diarias de las dos especies estudiadas son muy similares a las encontradas por Chatterjee \& Chandra (1997) para Gambusia affinis, especie conocida mundialmente por su capacidad de controlar larvas de culícidos. Esto denota la gran capacidad de consumo de las dos especies estudiadas y lo innecesarias que serían las introducciones de peces exóticos como controladores biológicos de las poblaciones de mosquitos en la región.

Por último, vale aclarar que López CAzorla et al. (2003) y QuinTANs et al. (2009) encuentran entre los ítems consumidos por $J$. multidentata especies que también son depredadoras de larvas de culícidos (como odonatos y decápodos), no presentes en los tractos digestivos de $C$. decemmaculatus, lo que podría ser negativo en el rol de controlador, como ha indicado BlAUSTEIN (1992) para $G$. affinis.

En el presente trabajo, además, se demostró una gran preferencia de las hembras de $C$. decemmaculatus hacia las larvas de A aegypti por sobre las de C. pipiens. Las causas de esta preferencia podrían ser el mayor tamaño de las partes quitinosas de las larvas de C. pipiens, lo que haría menos apetecibles a estas larvas con respecto a las otras, $\mathrm{y}$ el comportamiento más bentónico de las larvas de $A$. aegypti que, al respirar oxígeno atmosférico, tienen que atravesar la columna de agua con mucho más frecuencia que las otras larvas, lo que las deja más expuestas a la depredación por parte de los peces. RaJAzekHARAN \& ChOWDAiah (1972) registraron en G. affinis, una preferencia por larvas de $A$. aegypti por sobre las de $C$. fatigans y atribuyeron dicha preferencia por la primera especie a su menor tamaño (en las experiencias del presente trabajo no había diferencias en las longitudes totales de las especies de larvas), a su posición en la columna de agua (similar a lo observado en las experiencias del presente trabajo) y a la tendencia a formar grupos (no se observaron diferencias en los agrupamientos de las dos especies de larvas en este estudio). Lo anterior, nos lleva a pensar que la preferencia por larvas de $A$. aegypti estaría determinada por características comportamentales y morfológicas de A. aegypti y no debida a una particularidad de C. decemmaculatus.

A partir del presente trabajo se puede concluir que: en condiciones de laboratorio ambas especies de peces tienen la capacidad de alimentarse de larvas de C. pipiens. Jenynsia multidentata consume mayores cantidades de larvas de C. pipiens en periodos breves de tiempo con respecto a $C$. decemmaculatus. Los machos de $C$. decemmaculatus consumen menores cantidades de larvas de C. pipiens del $4^{\circ}$ estadío, en comparación con hembras de su misma especie y con $J$. multidentata de ambos sexos. Los consumos de larvas de $C$. pipiens en 24 horas por gramo de pez son muy similares para las especies estudiadas. El ancho de boca de las especies estudiadas tiene una relación importante con el número de larvas de $C$. pipiens que son capaces de consumir. Por último, las hembras de $C$. decemmaculatus tienen una fuerte preferencia por las larvas de $A$. aegypti por sobre las de $C$. pipiens. Por todo esto, las dos especies de peces estudiadas demostraron tener capacidades suficientes como para ser tenidas en cuenta en planes de manejo de poblaciones de mosquitos, evitándose la introducción de especies exóticas potencialmente invasoras con todos los perjuicios que esto conlleva.

Agradecimientos. A la Dra. Andrea Cecilia Hued, la Dra. María de los Angeles Bistoni y la Dra María Laura Ballesteros por la lectura crítica del manuscrito.

\section{REFERENCIAS BIBLIOGRÁFICAS}

Ayala, J. R.; Rader, R. B.; Belk, M. C. \& Schaajle, G. B. 2007. Groundtruthing the impact of invasive species spatio-temporal overlap between native least chub and introduced western mosquitofish. Biological Invasions 9(7):857-869.

Bistoni, M. A.; Hued, A.; Videla, M. \& SAgretti, L. 1999. Water quality effects on fish communities of the central part of Argentina. Revista Chilena de Historia Natural 72:325-335.

Blaustein, L. 1992. Larvivorous fishes fail to control mosquitoes in experimental rice plots. Hydrobiologia 232:219-232.

Chandra, G.; Bhattacharjee, I. \& Chatterjee, S. N. 2010. A review on Anopheles subpictus Grassi - A biological vector. Acta Tropica 115:142-154.

Chatterjee, S. N. \& Chandra, G. 1997. Laboratory trials on the feeding pattern of Anopheles subpictus, Culex quinquefasciatus and Armigeres subalbatus larvae by Gambusia affinis. Science and Culture 63:5152.

Gamradt, S. C. \& Kats, L. B. 1996. The effects of introduced crayfish and mosquito fish on California newts (Taricha torosa). Conservation Biology 10:1155-1162.

Giri, F. \& Collings, P. 2003. Evaluación de Palaemonetes argentinus (Decapoda, Natantia) en el control biológico de larvas de Culex pipiens (Diptera, Culicidae) en condiciones de laboratorio. Iheringia, Série Zoologia 93(3):237-242. 
Goodsell, J. A. \& Kats, L. B. 1999. Effect of introduced mosquito fish on Pacific tree frogs and the role of alternative prey. Conservation Biology 13:921-924.

Howe, E.; Howe, C.; Lim, R. \& Burchett, M. 1997. Impact of the introduced poecilid Gambusia holbrooki (Girard, 1859) on the growth and reproduction of Pseudomugil signifer (Kner, 1865) in Australia. Marine and Freshwater Research 48:425-434.

Hued, A. C. \& Bistoni, M. A. 2005. Development and validation of a Biotic Index for evaluation of environmental quality in the central region of Argentina. Hidrobiología 543:279-298.

Kumar, R. \& Hwang, J. S. 2005. Larvicidal Efficiency of Aquatic Predators: A Perspective for Mosquito Biocontrol. Zoological Studies 45:447-466.

Leyse, K. E.; Lawler, S. P. \& Strange, T. 2004. Effects of an alien fish, Gambusia affinis, on an endemic California fairy shrimp, Linderiella occidentalis: implications for conservation of diversity in fishless waters. Biological Conservation 118:57-65.

López Cazorla, A.; Durán, W. \& Tejera, L. 2003. Alimentación de la ictiofauna del Río Sauce Grande, Provincia de Buenos Aires, Argentina. Biología Acuática 20:73-79.

López, H. L.; Menni, R. C.; Donato, M. \& Miquelarena, A. M. 2008. Biogeographical revision of Argentina (Andean and Neotropical Regions): an analisis using freshwater fishes. Journal of Biogeography 35:1564-1579.

Marti, G. A.; Azpelicueta, M. M.; Tranchida, M. C.; Pelizza, S. A. \& GARCíA, J. J. 2006. Predation efficiency of indigenous larvivorous fish species on Culex pipiens L. larvae (Diptera: Culicidae) in drainage ditches in Argentina. Journal of Vector Ecology 31(1):102-106.

Quintans, F.; Scasso, F.; Loureiro, M. \& Yafe, A. 2009. Diet of Cnesterodon decemmaculatus (Poeciliidae) and Jenynsia multidentata (Anablepidae) in a hypertrophic shallow lake of Uruguay. Iheringia, Série Zoologia 99(1):99-105.
Quintans, F.; Scasso, F. \& Defeo, O. 2010. Unsuitability of Cnesterodon decemmaculatus (Jenyns, 1842) for mosquito control in Uruguay: evidence from food-preference experiments. Journal of Vector Ecology 35(2):333-338.

Rajazekharan, P. T. \& Chowdaiah, B. N. 1972. Selective Feeding Behavior of Gambusia affinis. Oecologia 11:79-81.

Ringuelet, R. A.; Aramburu, R. H. \& de Aramburu, A. A. 1967. Los peces argentinos de agua dulce. La Plata, Comisión de Investigaciones Científicas de la Provincia de Buenos Aires. 602p.

Rossi, G. C.; Mariluis, J. C.; Schnack, J. A. \& Spinelli, G. R. 2002. Dípteros vectores (Culicidae y Calliphoridae) de la Provincia de Buenos Aires. COBIOBO No 4, PROBIOTA n ${ }^{\circ} 3,45$.

Rowe, D. K.; Smith, J. P. \& Baker, C. 2007. Agonistic interactions between Gambusia affinis and Galaxias maculatus: implications for whitebait fisheries in New Zealand rivers. Journal of Applied Ichthyology 23(6):668-674.

Scasso, F.; Mazzeo, N.; Gorga, J.; Kruk, C.; Lacerot, G.; Clemente, J.; FABIÁN, D. \& Bonilla, S. 2001. Limnological changes of a subtropical shallow hypertrophic lake during its restoration. Two years of wholelake experiment. Aquatic Conservation: Marine Freshwater Ecosystems 11:31-44.

Tranchida, M. C.; Pelizza, S. A.; Bisaro, V.; Beltrán, C.; García, J. J. \& Micieli, M. V. 2010. Use of the neotropical fish Cnesterodon decemmaculatus for long-term control of Culex pipiens L. in Argentina. Biological Control 53:183-187.

Vezzani, D. \& Albicócco, A. P. 2009. The effect of shade on the container index and pupal productivity of the mosquitoes Aedes aegypti and Culex pipiens breeding in artificial containers. Medical and Veterinary Entomology 23:78-84. 